\title{
Results of a Pilot Randomized Placebo-Controlled Trial in Primary and Secondary Raynaud's Phenomenon with St. John's Wort: Detecting Changes in Angiogenic Cytokines When RP Improves
}

\author{
Déanne Malenfant, ${ }^{1}$ Kelly Summers, ${ }^{2,3}$ Shannon Seney, ${ }^{2}$ Donna McBain, ${ }^{4}$ Lisa Petrlich, \\ Sharon Watson, ${ }^{4}$ Louise Vanderhoek, ${ }^{4}$ Nooshin Samadi, ${ }^{1,4}$ Ashley Bonner, ${ }^{5}$ and Janet Pope ${ }^{1,4}$
}

\author{
${ }^{1}$ Department of Medicine, University of Western Ontario, London, ON, Canada N6A 5C1 \\ ${ }^{2}$ Screening Laboratory for Immune Disorders, Lawson Health Research Institute, London, ON, Canada N6A 4V2 \\ ${ }^{3}$ Department of Microbiology \& Immunology, University of Western Ontario, London, ON, Canada N6A 5C1 \\ ${ }^{4}$ Rheumatology, St. Joseph's Health Care London, London, ON, Canada N6A 4V2 \\ ${ }^{5}$ Department of Mathematics and Statistics, McMaster University, Hamilton, ON, Canada L8S 4K1
}

Correspondence should be addressed to Janet Pope, janet.pope@sjhc.london.on.ca

Received 6 May 2011; Accepted 27 June 2011

Academic Editors: M. G. Danieli, B. A. Eberhard, C. G. Mackworth-Young, and C.-H. Suh

Copyright (C) 2011 Déanne Malenfant et al. This is an open access article distributed under the Creative Commons Attribution License, which permits unrestricted use, distribution, and reproduction in any medium, provided the original work is properly cited.

\begin{abstract}
Objectives.To perform a 6-week double-blind RCT in Raynaud's phenomenon (RP) comparing the plant extract St. John's Wort (SJW) to placebo. Methods. RP patients having at least 7 attacks per week were stratified by primary and secondary RP and within secondary by systemic sclerosis or other connective tissue disease. Subjects completed a daily standardized diary recording all RP attacks (frequency, duration and severity). Serum levels of 18 inflammatory and angiogenic cytokines were measured pre- and post-treatment. Results. Eighteen patients completed the study; 8 received SJW and 10 placebo. The decrease in mean number of attacks per day was 0.75 with SJW and 1.01 with placebo, $P=0.06$. Attack duration and severity were not different between groups. Cytokine analyses demonstrated no between-groups differences. Combining treatment groups, those with $>50 \%$ improvement in frequency of attacks yielded a significant increase in E-selectin $(P=0.049)$, MMP-9 $(P=0.011)$, G-CSF $(P=0.02)$, and VEGF $(P=0.012)$ pre- versus post-treatment. $\mathrm{A} \geq 50 \%$ improvement in severity of attacks corresponded to a significant increase in levels of sVCAM-1 $(P=0.003)$, sICAM-1 $(P=0.007)$, and MCP-1 $(P=0.004)$. Conclusions. There were no clinical or biomarker benefit of SJW versus placebo in RP. However, combining all patients, there were changes in some cytokines that may be further investigated.
\end{abstract}

\section{Introduction}

Raynaud's phenomenon (RP) affects 3-5\% of the population [1] and is characterized by episodic vasospasm of digital arteries causing pallor, cyanosis, and/or rubor upon rewarming. It is caused by poor blood flow to digits, often occurring with cold exposure and stress. The severity of symptoms ranges from mild to severe pain, and in secondary $\mathrm{RP}$ there may be ischemic complications. RP can be primary (idiopathic) or secondary. Secondary RP is associated with an underlying disease (often a connective tissue disease $\{\mathrm{CTD}\}$ such as systemic sclerosis $\{\mathrm{SSc}$, scleroderma $\}$, systemic lupus erythematosus \{SLE\}, and Sjogren's syndrome).

The exact mechanisms causing RP are unknown, but inflammatory and angiogenic cytokines secreted by circulating cells and endothelial cells (ECs) may stimulate vascular abnormalities that ultimately reduce blood flow. Underlying endothelial dysfunction likely initiates and mediates this pathological process [2]. ECs in RP promote an inflammatory response through elevated production of proinflammatory cytokines [2] and increased expression of 
adhesion molecules $[3,4]$ and secretion of vasoconstrictor and profibrotic mediators, such as endothelin-1 and angiotensin, instead of vasodilators [5].

Primary RP is most often controlled with lifestyle changes (keeping warm, smoking cessation), whereas secondary RP in patients with SSc frequently requires systemic treatment. Depending on disease severity, the choice of medication includes calcium channel blockers (CCBs), such as nifedipine, and other vasodilators. CCBs in both primary and secondary Raynaud's have demonstrated modest benefit; however these medications frequently have side effects such as hypotension, flushing, peripheral edema, and headaches [6-8]. There is an unmet need for an effective and welltolerated treatment in moderate-to-severe RP.

One trial suggested that fluoxetine (an antidepressant) was effective in the treatment of both primary and secondary RP [9]. Fluoxetine is a selective serotonin reuptake inhibitor (SSRI) which in RP may be improving vascular endothelium [9]. Ketanserin, a selective antagonist of serotonin, has also been studied in RP [10]. Serotonin receptors have been shown to be important in vasospasm in patients with RP [11]. St. John's Wort (SJW, hypericum perforatum) is a natural herbal product with some SSRI-like properties. In mild-tomoderate depression, SJW appears to be equally effective, with fewer side effects than prescription SSRI drugs [1217]. Thus, SJW may be an effective, safe, and well-tolerated alternative treatment for RP via effects on serotonin. In this study, the dose of SJW was selected to be $600 \mathrm{mg}$ a day (given as $300 \mathrm{mg}$ BID). Depression trials have dosed SJW from 300 to as high as $900 \mathrm{mg}$ BID [12-17], where $300 \mathrm{mg}$ BID was superior to placebo and well tolerated $[16,17]$.

We performed a trial with SJW in subjects with primary and secondary RP. The hypothesis of this trial was that SJW would be more effective than placebo in the treatment of RP, as defined by decreased frequency, duration, and severity of attacks.

\section{Methods}

2.1. Study Design. This randomized, double-blinded, placebo-controlled trial was registered at clinicaltrials.gov.ca. It was approved by Health Canada (in the Natural Health Product division). Ethics approval was obtained from the University of Western Ontario Ethics Committee.

2.2. Subjects. Subjects were recruited from a rheumatology clinic over two winters, as Raynaud's symptoms are more severe at this time. All subjects had symptomatic RP and were stratified by primary versus secondary Raynaud's, and within secondary RP between systemic sclerosis and other connective tissue diseases. Inclusion criteria were primary or secondary RP as diagnosed by a rheumatologist, clinical need for treatment of RP with at least 7 attacks per week over a two-week run in, age at least 16 years, able to provide informed consent, and ability to complete the daily standardized RP symptom diary prior to randomization. The exclusion criteria consisted of a prior allergic reaction to SJW, depression requiring treatment, use of SSRIs or other antidepressants with the exception of low-dose amitriptyline used for reasons other than depression, use of drugs that may interact with SSRIs such as cyclosporine, warfarin, digoxin, and theophylline, clinically significant noncompliance, and anticipated need for surgery (sympathectomy) within 3 months of the trial. Subjects that met these criteria were randomized using computer-based randomization $1: 1$ to receive either SJW dosed at $300 \mathrm{mg}$ twice daily (BID) or a lactose placebo BID for 6 weeks. St. John's Wort (SJW) was provided by Webber Pharmaceuticals. SJW was reencapsulated by our research pharmacists who also made the encapsulated placebo. Subjects completed a daily journal with standardized questions about each attack (severity and duration). Using the diary, the number of attacks per day was calculated. They also recorded any potential adverse events (AEs). Every 2 weeks subjects were seen by a study nurse and asked about side effects and digital ulcers, and patient global assessments were performed.

Blood was drawn into EDTA tubes at 0 and 6 weeks posttreatment, immediately spun, and plasma frozen at $-80^{\circ} \mathrm{C}$ for later analysis of 18 inflammatory and angiogenic cytokines using multiplexed immunoassays (Millipore Corp., MA) and ELISA for TGF- $\beta 1$ (BD Biosciences, NJ).

2.3. Efficacy Measures. Primary outcome measures were the frequency of RP (number of attacks daily), severity of RP on a 0 to 10 scale, duration of RP attacks (in minutes) (from the RP diary), and changes in the levels of inflammatory and angiogenic cytokines between baseline and 6 weeks post-treatment. Secondary outcome measures assessed at the beginning and end of the treatment period were the health assessment questionnaire (HAQ) disability index, patient global assessment of RP, and the presence and location of digital ulcers.

2.4. Sample Size Calculation. Using a between-group difference in number of RP attacks at the end of the study of 2.4 attacks per day in placebo and a $45 \%$ reduction in attacks in SJW, and assuming an equal SD of 0.75 with $80 \%$ power and a two-sided $P$ value of 0.05 , we would need only 8 patients per group to complete the study. This was an early phase study (the first of SJW in RP), and the data were to direct future potential RP trials with SJW.

2.5. Statistics. Between-group comparisons (SJW compared to placebo) were done using 2-tailed, paired $t$-tests to analyze for significant changes (fold changes) in cytokine levels between baseline and 6 weeks post-treatment. Similar analyses were then performed combining all completers based on patient-reported improvement $(\geq$ or $<50 \%)$ in the frequency and severity of attacks, irrespective of treatment allocation, to find a correlation between cytokines and symptoms. Within-group comparisons were done using paired $t$-tests in completers to analyze for significant changes in the frequency, duration, and severity of attacks per day. 


\section{Results}

3.1. Subjects. Thirty-three patients with symptomatic RP were screened ( 30 females, 3 males) and 25 were randomized (as five had $<7$ attacks per week over the two week run in and three did not want to continue in the study). In those randomized, 5 had primary RP and 20 had secondary RP, of whom 11 had SSc and 2 were undifferentiated CTD, 2 SLE, 2 RA, and one each of mixed connective tissue disease, Sjogren's syndrome, and dermatomyositis. Eighteen subjects completed the trial ( 2 had primary RP and 8 with SSc), with 8 on active SJW and 10 on placebo. Adverse events (AEs) were common (57\% SJW, $43 \%$ placebo), especially headaches. While these AEs were generally mild, there was one serious $\mathrm{AE}$ in a subject with secondary RP and scleroderma that involved hospitalization due to atrial fibrillation. Seven who were randomized dropped out, 4 due to AEs ( 3 on SJW and one on placebo). There were no significant differences between SJW and placebo using ITT and also comparing only the completers.

3.2. Severity, Duration, and Frequency of Attacks. There were no significant differences in the severity, duration or frequency of attacks between the groups. However, there were significant within-group differences observed from beginning to end of the trial in both SJW and placebo groups (Table 1 shows the completers only analysis). There were no important differences for the secondary endpoints when comparing SJW to placebo.

The within-group pre- and post-treatment frequency and severity of attacks were not significant in the SJW group. In contrast, within the placebo group, the frequency of attacks per day decreased significantly $(P=0.001)$ as did the severity of attacks $(P=0.004)$. The duration of daily RP attacks decreased significantly with SJW $(P=0.002)$ and also with placebo $(P=0.009)$. The absolute change was numerically less with SJW than placebo. This difference in duration of attacks was further analyzed within the SJW group by the subtype of secondary RP (SSc and other CTD), but not in primary RP as the number of participants was too small. These analyses showed a significant improvement $(P=0.033)$ in the duration of attacks in subjects with secondary RP in the nonscleroderma group.

3.3. Cytokines. There were no significant changes in cytokine levels from pre- to post-treatment between the SJW and placebo. All patients who completed the study were collated (combining SJW and placebo) based on patient-reported scores into $\geq$ or $<50 \%$ improvement in the frequency and then severity of RP attacks to analyze the significance between cytokines and symptoms, irrespective of treatment allocation. Cytokines were analyzed as the percent change relative to baseline and also as mean differences between the start and end of treatment since both types of analyses may have biological relevance.

3.4. Percent Change. We found that those with a $\geq 50 \%$ improvement in patient-reported frequency of attacks was significantly associated with a larger fold increase in levels of MMP-9 $(P=0.027)$, MIP-1 $\beta(P=0.045)$, and VEGF $(P=0.002)$ between baseline and the end of treatment when compared to a $<50 \%$ improvement (data not shown). There was no significant correlation between severity of attacks and percent change in cytokine levels.

3.5. Mean Differences. Comparing subjects who improved by at least $50 \%$ to those who did not with respect to the frequency of RP attacks demonstrated significant changes in E-selectin $(P=0.049)$, MMP-9 $(P=0.011)$, G-CSF $(P=0.02)$, and VEGF $(P=0.012)$ (Table 2$)$. Using the same comparison for $\geq$ or $<50 \%$ improvement in severity of attacks, a significant increase in sVCAM-1 $(P=0.003)$, sICAM-1 $(P=0.007)$, and MCP-1 $(P=0.004)$ was observed (Table 3).

\section{Discussion}

SJW was not superior to placebo, and in fact, placebo was numerically but not significantly better than SJW on some outcomes. It is likely that shared properties between SSRIs and SJW in the treatment of depression are not similar in RP treatment at the dose studied [9]. SJW should not be pursued for the treatment of RP in a larger trial as there was no biomarker or clinical change that was superior to the placebo group.

There were significant within-group results which underscores that any open-label treatment trial needs to be interpreted with caution in RP as there is likely a large placebo effect or regression to the mean. Even proof-ofconcept trials testing new agents in RP should have a doubleblinded, randomized trial design.

There are multiple potential reasons why this is a negative trial which could include inefficacy of SJW, underdosing, heterogeneity of the patients, small sample size, inadequate outcome measurements, or spurious results, but there was not even a trend of improvement compared to placebo. Many published trials have combined primary and secondary RP stratifying by type of RP, which we did. We tried to have a more homogenous group of patients to study by using only patients with a high frequency of RP attacks. Also, the majority of patients were secondary RP, so perhaps they are more difficult to treat than primary RP, and in this group the numbers are incredibly small to make any treatment conclusion within this small subset of completers. The SJW dose was at the lower end of what has been used for depression trials but nevertheless the dose we chose had demonstrated efficacy $[16,17]$. The Raynaud's Condition Score (a daily self-assessment questionnaire of RP on an ordinal scale) [18] was not collected within the diaries but at each visit patient global RP assessment was done. Biomarker data also did not demonstrate that SJW is effective in RP. Some biomarkers improved in patients whose RP was better, but they did not improve more in the SJW group compared to placebo. The placebo changes for RP outcomes in this study may have occurred as there is often regression to the mean in RP trials. For instance, when patients enroll in $\mathrm{RP}$ trials requiring a large number of attacks, they enter at a high level of RP activity which may decrease over time, 
TABLE 1: Within-group characteristics for frequency, duration, and severity of RP in SJW compared to placebo in the completers. There were no between-group differences.

\begin{tabular}{|c|c|c|c|c|c|c|c|c|}
\hline & $\begin{array}{l}\text { Baseline SJW } \\
\qquad(N=8)\end{array}$ & $\begin{array}{l}\text { 6-Week } \\
\text { Followup } \\
\text { SJW }\end{array}$ & $\begin{array}{c}\text { Mean } \\
\text { difference } \\
\text { SJW } \\
\end{array}$ & $\begin{array}{c}P \text {-value } \\
\text { Within group } \\
\text { SJW }\end{array}$ & $\begin{array}{l}\text { Baseline } \\
\text { Placebo } \\
(N=10)\end{array}$ & $\begin{array}{l}\text { 6-Week } \\
\text { Followup } \\
\text { Placebo } \\
\end{array}$ & $\begin{array}{c}\text { Mean } \\
\text { difference } \\
\text { Placebo } \\
\end{array}$ & $\begin{array}{c}P \text {-value } \\
\text { Within group } \\
\text { Placebo } \\
\end{array}$ \\
\hline $\begin{array}{l}\text { Frequency (mean } \\
\text { no. of attacks per } \\
\text { day) }\end{array}$ & 3.175 & 2.4238 & .7513 & 0.1238 & 2.366 & 1.353 & 1.0130 & 0.001 \\
\hline $\begin{array}{l}\text { Duration (mean } \\
\text { total of attacks in } \\
\text { minutes per day) }\end{array}$ & 28.279 & 18.009 & 10.2703 & 0.0019 & 26.010 & 13.510 & 12.4992 & 0.009 \\
\hline $\begin{array}{l}\text { Severity (scale } 0 \text { to } \\
10 \text { ) }\end{array}$ & 4.752 & 3.785 & .9668 & 0.0758 & 2.954 & 1.947 & 1.0066 & 0.004 \\
\hline
\end{tabular}

TABLE 2: Within-group comparisons of patient-reported improvements in the frequency of Raynaud's attacks and the difference of cytokine levels between pre- and post-treatment. $P$ values were calculated using 2 -tailed, paired $t$-tests. Data represents mean comparisons of changes in cytokines in those who had more than $50 \%$ reduction in frequency (number) of RP attacks compared to those who did not.

\begin{tabular}{|c|c|c|c|c|c|}
\hline \multirow[t]{2}{*}{ Cytokine } & \multicolumn{2}{|c|}{ Mean cytokine change } & \multirow[t]{2}{*}{ Difference } & \multirow[t]{2}{*}{ Std. error difference } & \multirow[t]{2}{*}{$P$ value } \\
\hline & $\begin{array}{l}\text { Frequency of attacks } \\
\text { improved by } \geq 50 \% \\
(n=7)\end{array}$ & $\begin{array}{l}\text { Frequency of attacks } \\
\text { improved by }<50 \% \\
(n=6)\end{array}$ & & & \\
\hline sE-Selectin & 5843.8 & -3473.3 & 9317.1 & 4203.1 & 0.049 \\
\hline sVCAM-1 & 134550.6 & -14819.9 & 149370.5 & 122138.5 & 0.247 \\
\hline sICAM-1 & 8861.6 & -13292.5 & 22154.1 & 17813.4 & 0.239 \\
\hline MMP-9 & 68574.0 & -104997.8 & 173571.8 & 57260.1 & 0.011 \\
\hline tPAI-1 & -188.4 & -10086.1 & 9897.7 & 7392.6 & 0.208 \\
\hline IL-4 & -0.2 & 0.0 & -0.2 & 0.2 & 0.356 \\
\hline IL-6 & -2.6 & 0.1 & -2.7 & 2.9 & 0.387 \\
\hline IL-8 & -0.4 & -1.1 & 0.7 & 1.9 & 0.726 \\
\hline IL-10 & 0.0 & 0.0 & & & \\
\hline IL-12p70 & 0.3 & 0.1 & 0.3 & 0.3 & 0.416 \\
\hline IL-17 & 0.0 & -0.5 & 0.5 & 0.5 & 0.363 \\
\hline Eotaxin & -4.6 & 0.6 & -5.2 & 9.4 & 0.588 \\
\hline Basic FGF & 1.0 & 3.4 & -2.5 & 2.9 & 0.408 \\
\hline$G-C S F$ & 15.0 & -12.1 & 27.0 & 10.0 & 0.020 \\
\hline IFN- $\gamma$ & -13.6 & 0.6 & -14.1 & 21.8 & 0.531 \\
\hline IP-10 & 110.1 & 78.9 & 31.2 & 106.8 & 0.776 \\
\hline MCP-1 & -12.4 & -3.1 & -9.3 & 20.2 & 0.654 \\
\hline MIP- $1 \alpha$ & -0.4 & 0.3 & -0.7 & 0.5 & 0.199 \\
\hline MIP- $1 \beta$ & 7.1 & -3.5 & 10.6 & 5.5 & 0.082 \\
\hline PDGF-BB & -133.4 & -244.1 & 110.7 & 309.2 & 0.727 \\
\hline TNF- $\alpha$ & -1.0 & 2.2 & -3.2 & 3.1 & 0.341 \\
\hline$V E G F$ & 7.3 & -11.5 & 18.8 & 6.3 & 0.012 \\
\hline TGF- $\beta 1$ & -2.4 & -4.1 & 1.7 & 2.9 & 0.565 \\
\hline
\end{tabular}

and attacks are often related to cold temperature, which may vary over the study as a 6-week long trial started in winter may finish for some patients in spring. However, we cannot infer that SJW was worse than placebo as there were no statistically significant between-group differences and in both allocations there was improvement of RP. The study is too small to comment on individual background medications but patients were in a steady state if they were using calcium channel blockers or losartan for RP. We did not stop a partially effective drug due to the ethics of stopping treatment during winter in patients with more than 7 attacks per week on their current treatment if it was perceived to have partial benefit. We did not find lack of tolerability with other RP medications when adding SJW, but the numbers are too small. SJW does have many potential drug interactions but the Health Canada-approved protocol excluded only 
TABLE 3: Within-group comparisons of patient-reported improvements in the severity of Raynaud's attacks and the difference of cytokine levels between pre- and post-treatment. $P$ values were calculated using 2 -tailed, paired $t$-tests.

\begin{tabular}{|c|c|c|c|c|c|}
\hline \multirow[t]{2}{*}{ Cytokine } & \multicolumn{2}{|c|}{ Mean cytokine change } & \multirow[t]{2}{*}{ Mean difference } & \multirow[t]{2}{*}{ Std. error difference } & \multirow[t]{2}{*}{$P$ value } \\
\hline & $\begin{array}{l}\text { Severity of attacks } \\
\text { improved by } \geq 50 \% \\
\quad(n=2)\end{array}$ & $\begin{array}{c}\text { Severity of attacks } \\
\text { improved by }<50 \% \\
(n=11)\end{array}$ & & & \\
\hline sE-Selectin & 1429.1 & 1564.4 & -135.3 & 6985.0 & .985 \\
\hline sVCAM-1 & 443639.4 & -3122.2 & 446761.6 & 119191.6 & .003 \\
\hline sICAM-1 & 27421.2 & -6596.9 & 34018.1 & 10030.8 & .007 \\
\hline MMP-9 & 31051.5 & -19279.3 & 50330.7 & 106102.6 & .645 \\
\hline tPAI-1 & 1203.7 & -5840.2 & 7043.9 & 10808.5 & .528 \\
\hline IL-4 & 0.0 & -0.2 & 0.2 & 0.4 & .689 \\
\hline IL-6 & 0.0 & -1.6 & 1.6 & 4.5 & .724 \\
\hline IL-8 & 1.4 & -1.2 & 2.6 & 2.4 & .305 \\
\hline IL-10 & 0.0 & 0.0 & & & \\
\hline IL-12p70 & -0.1 & 0.2 & -0.3 & 0.4 & .447 \\
\hline IL-17 & 0.0 & -0.3 & 0.3 & 0.7 & .689 \\
\hline Eotaxin & 4.0 & -3.3 & 7.3 & 12.9 & .582 \\
\hline Basic FGF & -1.0 & 2.7 & -3.7 & 3.9 & .363 \\
\hline G-CSF & 8.8 & 1.3 & 7.5 & 17.7 & .680 \\
\hline IFN- $\gamma$ & 9.0 & -10.0 & 19.0 & 30.2 & .542 \\
\hline IP-10 & 188.5 & 78.8 & 109.7 & 144.4 & .463 \\
\hline$M C P-1$ & 27.8 & -14.7 & 42.4 & 11.4 & .004 \\
\hline MIP- $1 \alpha$ & 0.0 & 0.0 & 0.0 & 0.8 & .956 \\
\hline MIP- $1 \beta$ & 3.1 & 2.1 & 1.0 & 8.8 & .916 \\
\hline PDGF-BB & 3.0 & -218.6 & 221.6 & 424.4 & .612 \\
\hline TNF- $\alpha$ & -0.4 & 0.7 & -1.1 & 4.2 & .798 \\
\hline VEGF & 9.5 & -3.4 & 12.9 & 11.0 & .265 \\
\hline TGF- $\beta 1$ & -3.5 & -3.1 & -0.4 & 4.1 & .919 \\
\hline
\end{tabular}

drug-SJW interactions which were thought to be of clinical relevance.

AEs were common in this trial, and one SAE in a patient using SJW occurred. Since our incidence of AEs was fairly similar in SJW and placebo, many side effects were likely not related to the study medication.

Patients that reported a $\geq 50 \%$ improvement in the frequency of Raynaud's attacks were associated with higher levels of the proangiogenic cytokines, MMP-9, MIP- $1 \beta$, sEselectin, G-CSF, and VEGF. Similarly, decreased severity of these attacks corresponded to increases in plasma levels of sVCAM-1, sICAM-1, and MCP-1. We did not correct for multiple tests, and some of the changes may be due to withinpatient variability of the cytokine levels. However, testing these cytokines may be useful in other proof-of-concept trials with different agents in RP to determine if any are associated with clinically relevant RP outcomes.

\section{Acknowledgments}

St. John's Wort was supplied by Webber Pharmaceuticals and gel coated by St. Joseph's pharmacy to make an identical placebo. In no way did Webber influence the conduct or results of the trial. D. Malenfant was a medical student who was funded by the Schulich Research Training Program (SRTP).

\section{References}

[1] L. G. Suter, J. M. Murabito, D. T. Felson, and L. Fraenkel, “The incidence and natural history of Raynaud's phenomenon in the community," Arthritis and Rheumatism, vol. 52, no. 4, pp. 1259-1263, 2005.

[2] R. Bakst, J. F. Merola, A. G. Franks, and M. Sanchez, "Raynaud's phenomenon: pathogenesis and management," Journal of the American Academy of Dermatology, vol. 59, no. 4, pp. 633-653, 2008.

[3] S. N. Lambova and U. Müller-Ladner, "New lines in therapy of Raynaud's phenomenon," Rheumatology International, vol. 29, no. 4, pp. 355-363, 2009.

[4] B. Kahaleh, O. Meyer, and R. Scorza, "Assessment of vascular involvement," Clinical and Experimental Rheumatology, vol. 21, no. 3, pp. S9-S14, 2003.

[5] S. Rajagopalan, D. Pfenninger, C. Kehrer et al., "Increased asymmetric dimethylarginine and endothelin 1 levels in secondary Raynaud's phenomenon," Arthritis and Rheumatism, vol. 48, no. 7, pp. 1992-2000, 2003.

[6] J. E. Pope, "The diagnosis and treatment of Raynaud's phenomenon: a practical approach," Drugs, vol. 67, no. 4, pp. 517-525, 2007. 
[7] A. E. Thompson and J. E. Pope, "Calcium channel blockers for primary Raynaud's phenomenon: a meta-analysis," Rheumatology, vol. 44, no. 2, pp. 145-150, 2005.

[8] A. E. Thompson, B. Shea, V. Welch, D. Fenlon, and J. E. Pope, "Calcium-channel blockers for Raynaud's phenomenon in systemic sclerosis," Arthritis and Rheumatism, vol. 44, no. 8, pp. 1841-1847, 2001.

[9] B. Coleiro, S. E. Marshall, C. P. Denton et al., "Treatment of Raynaud's phenomenon with the selective serotonin reuptake inhibitor fluoxetine," Rheumatology, vol. 40, no. 9, pp. 10381043, 2001.

[10] J. Pope, D. Fenlon, A. Thompson et al., "Systemic Sclerosis (SS): ketanserin versus placebo for the treatment of Raynaud's phenomenon in progressive SS," Cochrane Database of Systematic Reviews, no. 2, Article ID CD000954, 2000.

[11] A. L. Herrick, "Pathogenesis of Raynaud's phenomenon," Rheumatology, vol. 44, no. 5, pp. 587-596, 2005.

[12] K. Clement, C. R. Covertson, M. J. Johnson, and K. Dearing, "St. John's Wort and the treatment of mild to moderate depression: a systematic review," Holistic Nursing Practice, vol. 20, no. 4, pp. 197-203, 2006.

[13] Y. Lecrubier, G. Clerc, R. Didi, and M. Kieser, "Efficacy of St. John's Wort extract WS 5570 in major depression: a double-blind, placebo-controlled trial," American Journal of Psychiatry, vol. 159, no. 8, pp. 1361-1366, 2002.

[14] E. Schrader, "Equivalence of St John's Wort extract (Ze 117) and fluoxetine: a randomized, controlled study in mildmoderate depression," International Clinical Psychopharmacology, vol. 15, no. 2, pp. 61-68, 2000.

[15] V. Schulz, "Safety of St. John's Wort extract compared to synthetic antidepressants," Phytomedicine, vol. 13, no. 3, pp. 199-204, 2006.

[16] S. Kasper, I. G. Anghelescu, A. Szegedi, A. Dienel, and M. Kieser, "Superior efficacy of St John's Wort extract WS ${ }^{\circledR} 5570$ compared to placebo in patients with major depression: a randomized, double-blind, placebo-controlled, multi-center trial [ISRCTN77277298]," BioMed Central Medicine, vol. 4, p. $14,2006$.

[17] M. Mannel, U. Kuhn, U. Schmidt, M. Ploch, and H. Murck, "St. John's Wort extract LI160 for the treatment of depression with atypical features-a double-blind, randomized, and placebo-controlled trial," Journal of Psychiatric Research, vol. 44, no. 12, pp. 760-767, 2010.

[18] P. A. Merkel, K. Herlyn, R. W. Martin et al., "Measuring disease activity and functional status in patients with scleroderma and Raynaud's phenomenon," Arthritis and Rheumatism, vol. 46, no. 9, pp. 2410-2420, 2002. 


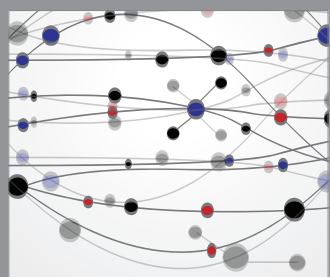

The Scientific World Journal
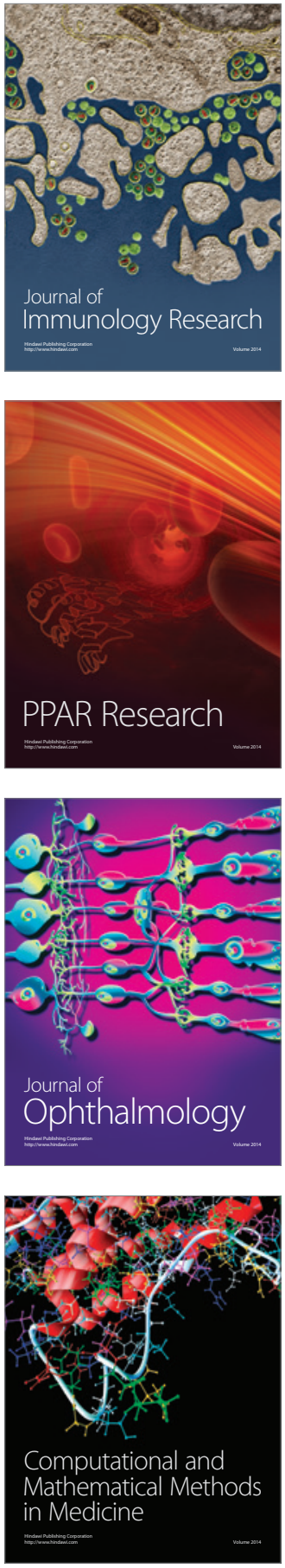

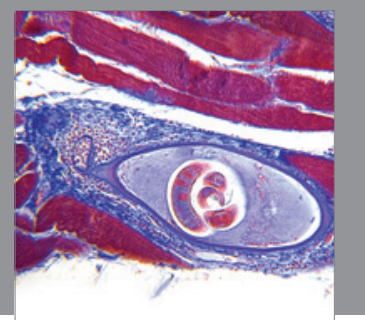

Gastroenterology

Research and Practice
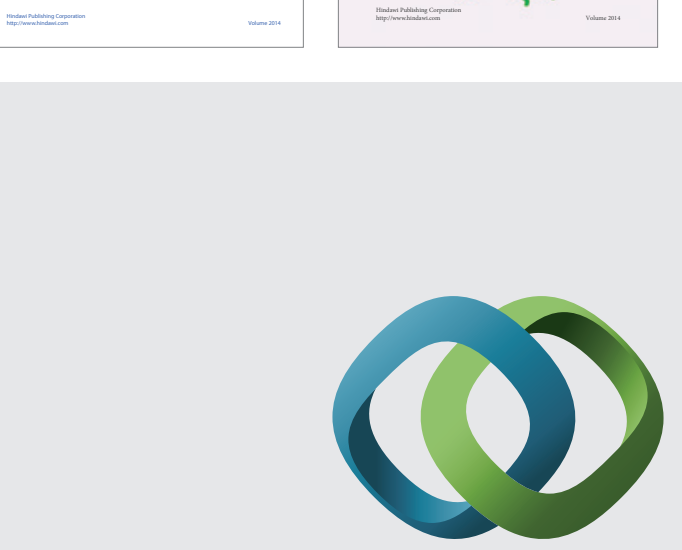

\section{Hindawi}

Submit your manuscripts at

http://www.hindawi.com
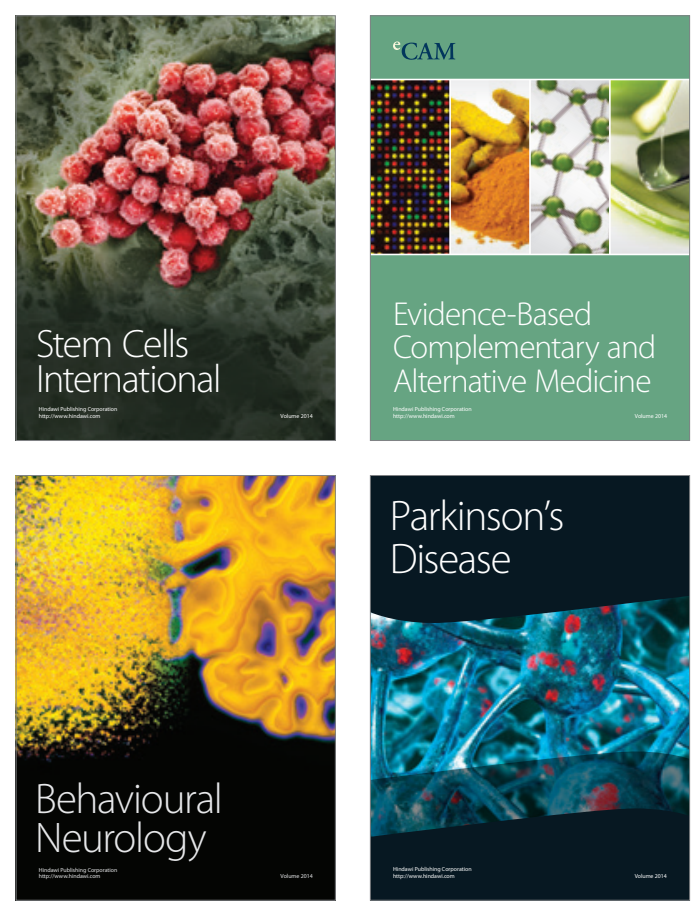

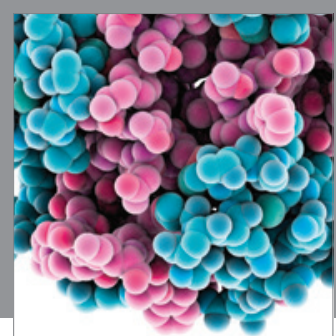

Journal of
Diabetes Research

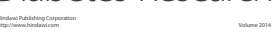

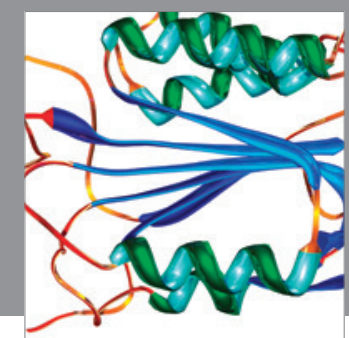

Disease Markers
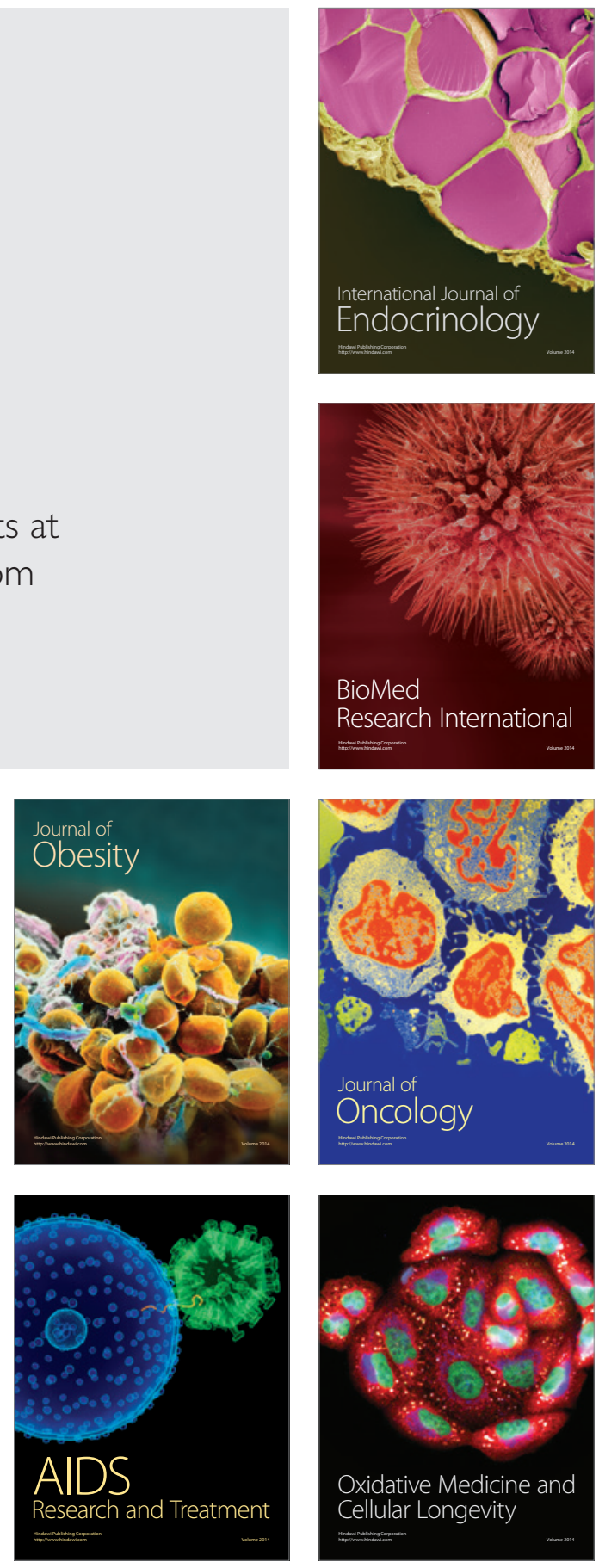